\title{
Acoustic Emissions analysis of a four-point bending test on a Reinforced Concrete beam
}

\author{
Corrado Chisari ${ }^{1}$, Claudio Guarnaccia ${ }^{1,}{ }^{*}$, Nicola Lamberti $^{2}$, Vincenzo Piluso ${ }^{1}$, Joseph $_{\text {Quartieri }}{ }^{1}$, and Gianvittorio Rizzano $^{1}$ \\ ${ }^{1}$ University of Salerno, Department of Civil Engineering, via Giovanni Paolo II, 132 - 84084 Fisciano (SA), Italy \\ ${ }^{2}$ University of Salerno, Department of Industrial Engineering, via Giovanni Paolo II, 132 - 84084 Fisciano (SA), Italy
}

\begin{abstract}
Assessment of existing structures by means of non-destructive techniques is an important research topic in modern engineering, because of its potential in estimating structural health status without introducing undesired damage. Among several recognized techniques, Acoustic Emission (AE) analysis is growing interest in the scientific community, since it can be used to detect the initiation of cracking processes, to determine cracking typology and to extract other information from the specimen. In this paper, the authors present the results of a four-point bending test on a Reinforce Concrete (RC) beam, performed at the University of Salerno. The test has been conducted according to a loading protocol characterised by several loading-unloading cycles, and both mechanical and acoustic data have been recorded. The results will be presented in terms of standard AE parameters, commonly adopted in literature, such as Felicity ratio, signal strength and cumulative signal strength, historic index and $b$ value. It will be underlined that these parameters allow to detect interesting moments of the phenomenon (cracking onset and development) and to assess the damage of the structure.
\end{abstract}

\section{Introduction}

The development of non-destructive tests for the damage assessment of Reinforced Concrete (RC) and other materials structures is arising interest among researchers and stakeholder. Many structures, such as bridges, buildings, tanks, etc., built in the past decades, increasingly show signs of decay and need to be monitored.

Real scale tests have been pursued by some of us, in the framework of seismic response of structures [1, 2]. On the other hand, FEM modelling has been performed by some of us, to evaluate damaging and breaking behaviour of steel and steel/concrete structures [3, 4].

Among the recognized non-destructive techniques, Acoustic Emissions (AE) is one of the most interesting to be studied and improved, since it is able to furnish several details on the status of the material and on the cracking pattern.

Several studies are present in literature, in which the $\mathrm{AE}$ analysis has been studied, mainly in laboratory tests. In [5], the authors investigate the possibility to use Acoustic Emission (AE) techniques to estimate the damage in single-edge notched beam tests. The Weibull damage function is assumed, based on earlier research, and it is calibrated onto the $\mathrm{AE}$ counts results. The comparison study indicated that the Weibull damage function calibrated from the acquired $\mathrm{AE}$ data can describe the damage response of both normal strength and high performance concrete. An interesting auxiliary procedure to determine the attenuation of $\mathrm{AE}$ signals due to the medium is also described.

[6] presents a review on the use of AE for structural health monitoring of concrete structures. Two approaches for the analysis of AE data are described: (i) parametric analysis, which makes use of parameters as counts, maximum amplitude, energy of the signals, and (ii) signal waveform analysis, which allows better noise discrimination, 3D localization and source mechanism identification. As approach (ii) requires a large amount of data to be stored and treatment of the data by specialised tools, it is less common than parametric analysis in the analysis of concrete structures. It must be pointed out that source localization may be achieved by means of parametric analysis also (by comparing arrival times of the signals), even though a number of shortcomings have been highlighted in [7], i.e. mode conversions, dispersion and attenuation of waves may result in sensors recording the arrival of different wave modes that have travelled with different velocities. Some authors [8] show that parametric analysis may also be used to reveal the type of cracking, i.e. tensile or shear cracking, based on rise time and frequency. At this stage, thus, it seems useful to focus on parametric analysis, which is simpler and requires less data to be stored. The most common use of AE data processed by means of parametric analysis, apart from the previously mentioned task of source localization and damage mechanism identification, is damage severity evaluation of existing structures. To this aim, [6] distinguishes five methods:

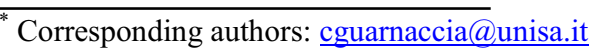


1. Correlation of calm and felicity ratio, exploiting the phenomenon of Kaiser effect [9]. This has been codified by the Japanese standard [10];

2. The use of b-value [11-14], and, more recently, by Carpinteri et al. [15], which proposed analytical correlation with theory of fracture [16] and numerical models [17] [18]. The approach of linking AE experimental data to numerical simulations seems interesting, and quite unexplored at this stage;

3 . Historic and severity indices $[19,20]$;

4. The use of relaxation ratio, which measure the release of AE energy during unloading [21];

5. The use of cumulative signal strength ratio [22], accounting for Kaiser and Felicity ratio and in positive relationship with the growth of the level of damage.

In this paper, the authors present the results of the analysis of $\mathrm{AE}$ measured during a four-point bending test on a Reinforced Concrete (RC) beam.

After presenting the main parameters of the analysis (section 2), the experimental setup, together with some details on the offline data processing, will be briefly described (section 3 ). In section 4 , the results of the analysis will be reported, with particular care to the parameters that exhibit the most interesting performances in terms of damage assessment.

\section{Parameter-based AE analysis}

\subsection{Single event parameters}

In the $\mathrm{AE}$ analysis described in this paper, the signals received from the piezo-electric sensors were recorded by an oscilloscope after threshold exceeding as voltage-time data. The generic basic parameters describing the features of a signal are qualitatively illustrated in Fig. 1.

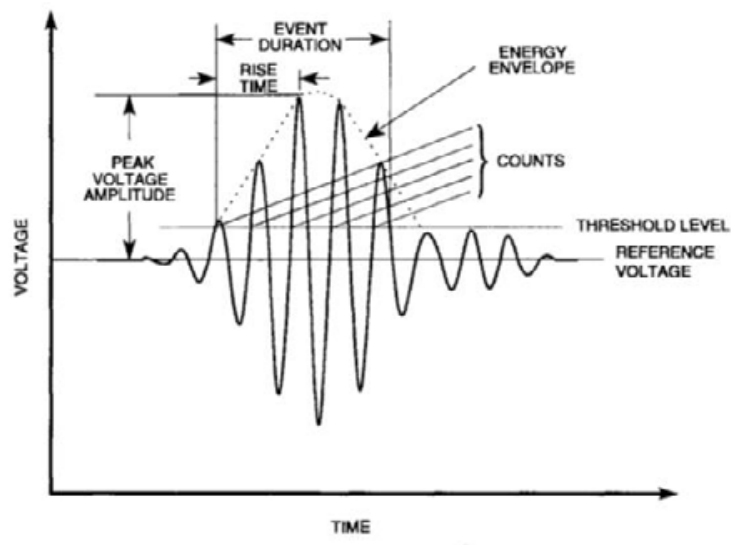

Fig. 1. Typical AE signal, with time-domain parameters [23].

From these we can define:

- Peak voltage amplitude (in V): $A_{\max }$;

- Peak voltage amplitude (in dB) [7]:

$$
A_{d B}=20 \log \left(\frac{A_{\max }}{A_{\text {ref }}}\right)-20 \log \left(g_{f}\right)
$$

where $g_{f}$ is the gain factor due to the amplifier, and $A_{\text {ref }}=1 \mu \mathrm{V}$ is a reference value.

- Duration $d=t_{f}-t_{i}$ (with $t_{i}, t_{f}=$ time of the first and

last threshold passing respectively), counts $c$, rise time $t_{r}$ and damping time $t_{d}$ (Fig. 1)

- Count rate: $c / d$;

- Count to peak: number of peaks from $t_{i}$ and $t\left(A_{\max }\right)$ maximum amplitude;

- Signal strength: $S=\int_{t=t_{i}}^{t_{f}} A_{\text {env }}(t) d t$ where $A_{\text {env }}(t)$ is the positive signal envelope.

\subsection{History parameters and $b$-value}

After reducing the data collected by the oscilloscope for the single event to an ensemble of synthetic parameters as those outlined in the previous subsection, damage assessment may be performed by studying the following parameters which account for the evolution of signal characteristics:

- Cumulative Signal Strength $\operatorname{CSS}(t)=\sum_{j=0}^{t} S_{j}$;

- Historic index [19]: $H(t)=\frac{N}{N-K} \frac{\sum_{i=K+1}^{N} s_{i}}{\sum_{i=1}^{N} S_{i}}$

where $K=\left\{\begin{array}{cc}0 & N>C_{1} \\ C_{3} N & C_{1} \leq N<C_{2} \\ N-C_{1} & N \geq C_{2}\end{array}\right.$

$N$ number of events occurred up to time $t$

$C_{1}, C_{2}, C_{3}$ numerical constants;

- Felicity ratio (or load ratio [10]), defined as the ratio of the load at the onset of AE activity in subsequent loading to the previous load;

- $b$-value.

It is observed by many authors that the cumulative distribution of event magnitudes $A_{d B}$ varies with increasing damage, and events of larger magnitude occur less frequently than events of smaller magnitude. In analogy with seismology, this statement is expressed by the Gutenberg-Richter relationship [13] [16]:

$$
\log _{10} N=a-b M_{L}
$$

where the magnitude $M_{L}$ is proportional to the logarithm of the maximum amplitude $A_{\max }, N$ is the cumulative number of events with magnitude $\geq M_{L}$ and $a, b$ parameters of the curve. From (1) and (2) it follows that:

$$
\log _{10} N=a-b^{\prime} A_{d B}
$$

and thus, in line with [13], $b=20 b^{\prime}$.

Some authors [16] suggest that, based on the fractal geometry approach in the mechanics of damage and fracture of heterogeneous materials, $b$-value should approach 1.0 with the evolution of damage. However, this conclusion is only partly supported by experimental observations [13, 24-27], while all authors report high fluctuation of the $b$-value during the test. 


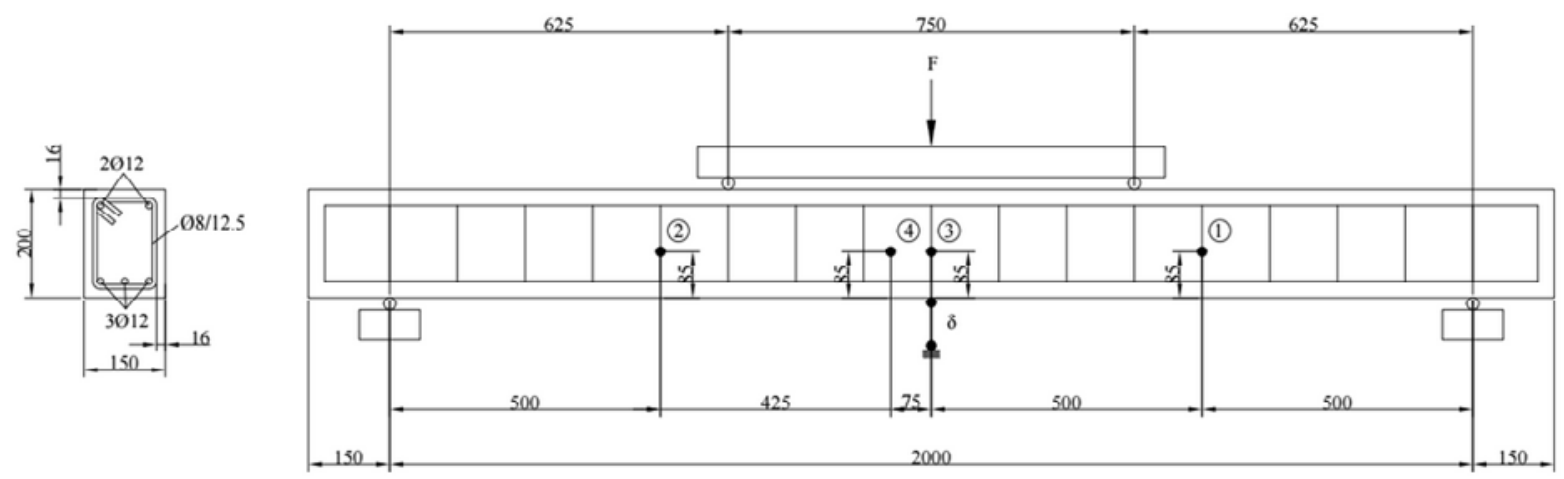

Fig. 2. Details of the test.

\section{The experimental test}

\subsection{Description of the test and measurement setup}

A four-point bending test on a reinforced concrete beam was performed at the Laboratory of Materials and Structures of the Department of Civil Engineering of the University of Salerno (Fig. 2). The simply supported, $150 \times 200 \mathrm{~mm}^{2}$ cross-section beam with length equal to $2000 \mathrm{~mm}$, was loaded in two points $625 \mathrm{~mm}$ far from the supports by means of a stiff steel beam transferring the load from a hydraulic jack (Fig. 3). The load was applied in displacement control, according to a protocol involving preliminary increasing cycles and then a ramp until failure.

Compressive tests on $150 \mathrm{~mm}$-deep concrete cubes provided a mean strength equal to $40.62 \mathrm{MPa}$, while tensile tests on steel bars resulted in mean yielding strength equal to $560.23 \mathrm{MPa}$.

The central unit recorded the total force $F$ transferred by the hydraulic jack and the displacement $\delta$ at midspan. Furthermore, four piezoelectric transducers, marked in Fig. 2 by a circle around the number, were placed on the front surface of the beam, $85 \mathrm{~mm}$ far from the bottom edge. Sensors 1 and 2 (see Fig. 2) have a 40 $\mathrm{kHz}$ resonance frequency; sensor 3 has a wider range of resonance $(40-80 \mathrm{kHz})$; sensor 4 is centred on higher frequencies (200 $\mathrm{kHz}$ resonance).

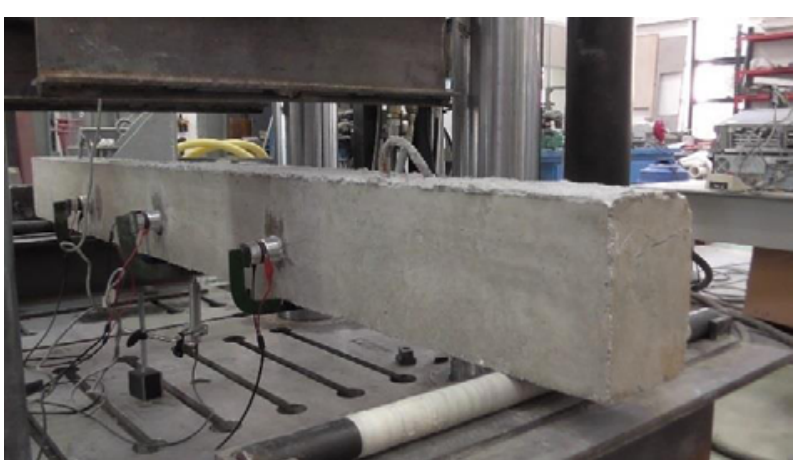

Fig. 3. View of the experimental setup.

The signals detected by sensors 1,2 and 3 were filtered and pre-amplified (factor 38 ) by a custom circuit. Signals from sensor 4 were not filtered and pre-amplified of a factor 10. All the signals were collected by a LeCroy oscilloscope, that stored data included in a $10 \mathrm{~ms}$ window from the pre-trigger. The trigger was set on sensor 3, i.e. the one supposed to be closest to the cracking region and that should have the highest signals. The instruments and the data acquisition have been provided by the personnel of the TAU lab of the University of Salerno.

The final load-displacement plot is shown in Fig. 4, together with the analytical values of first-cracking and ultimate loads, $F_{c r}=10.05 \mathrm{kN}$ and $F_{u}=91.97 \mathrm{kN}$ respectively. The former is evaluated considering the uncracked cross-section and tensile strength equal to 2.61MPa for concrete (estimated by means of Model Code [28] formulation starting from the compressive strength); conversely, $F_{u}$ was calculated according the usual hypotheses of reinforced concrete at the ultimate state, assuming a stress block approximation for the stress-strain relationship of concrete and elastic-plastic behaviour for steel.

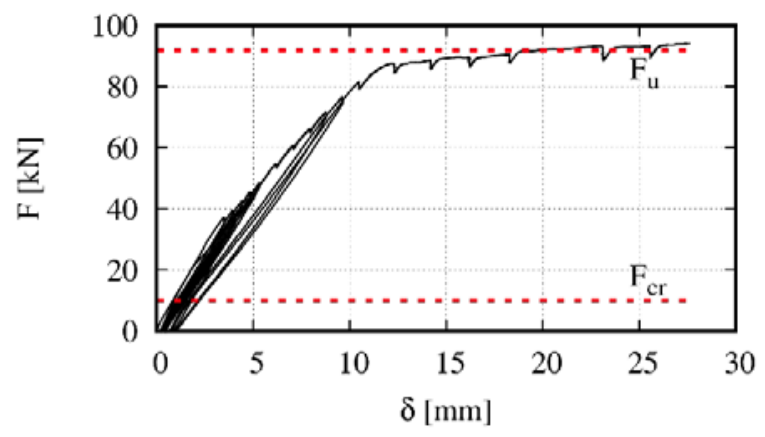

Fig. 4. Load-displacement plot in the four-point bending test.

\subsection{Offline data processing}

Any signal recorded was not used as-is in the parameterbased analysis, but underwent a cleaning process consisting of:

- pre-amplification removal (scaling);

- shifting of the signal to make median equal to zero (to remove low-frequency fluctuations which in the event window considered may be approximated as constant noise);

- setting any value under the threshold equal to zero.

The noise threshold is critical for the definition of many parameters but rather case-dependent. In this work, it was set as $0.01 \mathrm{~V}$. 


\section{Results}

\subsection{Felicity effect and cumulative signal strength}

For the sake of simplicity, only the data recorded by sensor 3, placed in the mid-span, will be discussed. The total number of events recorded was 1212, but after cleaning they were reduced to 414 only. In Fig. 5 the force and displacement histories are plotted as lines, while the events are highlighted as point markers. The grey vertical lines represent the attainment of 10, 20, $\ldots \%$ of total number of events.
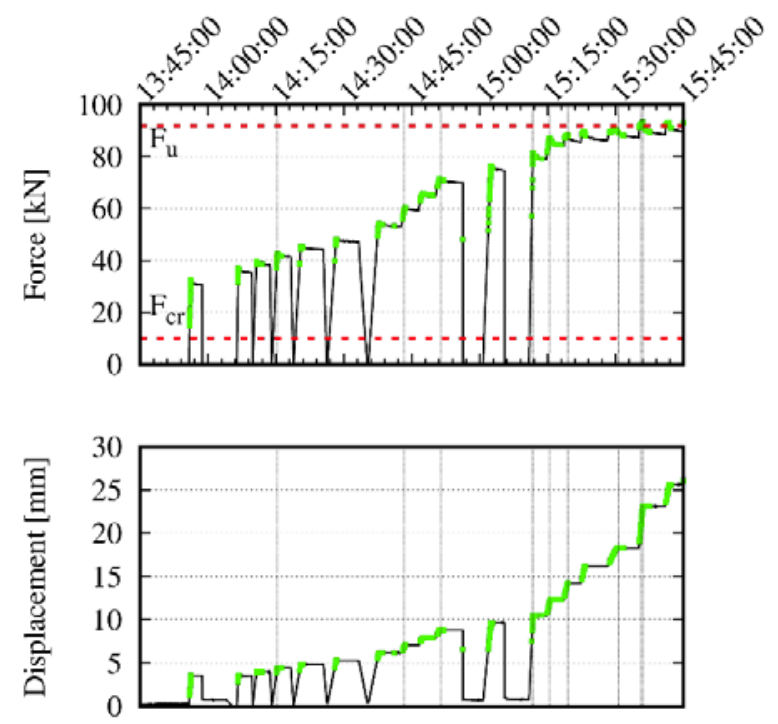

Fig. 5. Force and displacement histories.

Looking at Fig. 5, it is possible to observe that the first events, occurring when the lowest cross-section points reach concrete tensile strength, were recorded in correspondence of the evaluated $F_{c r}$ value. Furthermore, the Kaiser effect, stating that in undamaged conditions the acoustic emissions appear only when the maximum load previously attained is exceeded, is generally confirmed (Felicity ratio equal to 1 ). In the latest stages of the test (basically in the last two unloadingreloadings) the Felicity effect is conversely evident, and $\mathrm{AE}$ events are recorded for loads below the maximum load attained so far. No events were recorded in the unloading branches, except for a single event at 14:47.

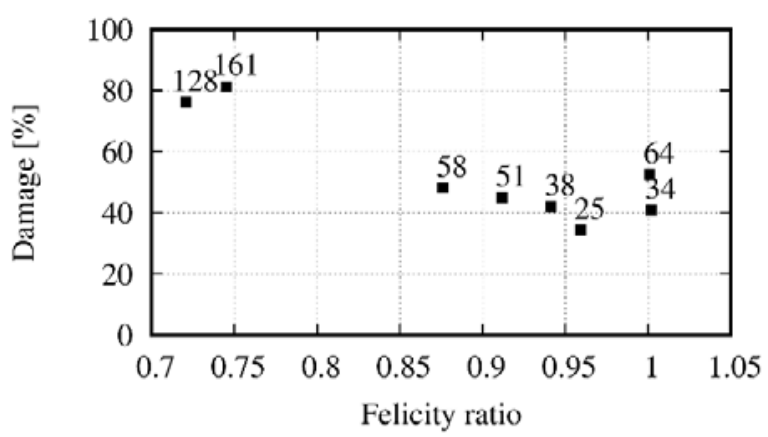

Fig. 6. Felicity effect as function of the damage (the numbers identify the events).
In Fig. 6, the Felicity ratio, evaluated for the eight unloading-reloading cycles performed in the test, is plotted against the damage $D$, defined in a simplified way here as:

$$
D=\frac{F_{\max }}{F_{u}}
$$

where $F_{\max }$ is the maximum load attained so far and $F_{u}$ is the ultimate load for the specimen, considering the whole test. Even though no clear trend is observable, it is evident that for damage under $60 \%$, the Felicity ratio is greater than 0.85 , while when the specimen is significantly damaged $(D>70 \%)$, the Felicity ratio is under 0.75. The threshold of 0.85 for damaged/undamaged specimens is similar to that reported in [29] and recommended in [30].

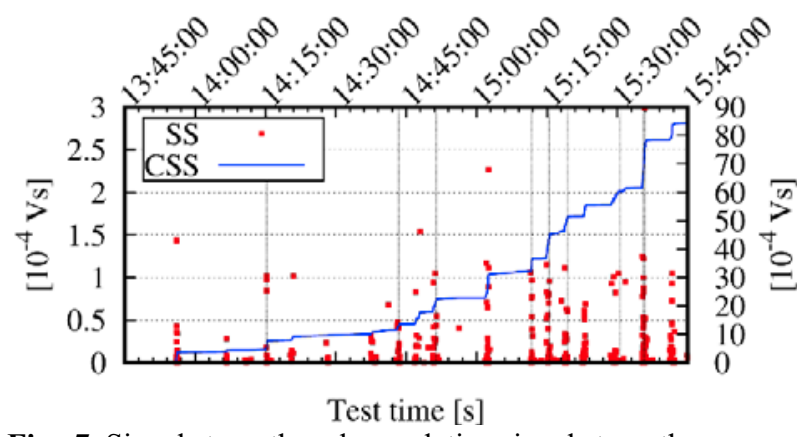

Fig. 7. Signal strength and cumulative signal strength.

In Fig. 7 signal strength and cumulative signal strength are plotted as function of time. It is possible to see that the most energetic events do not occur necessarily at the end of the test, i.e. in the damaged state, but may appear at the beginning. On the contrary, aggregated results show a clearer picture of the damage evolution, as the sudden increment in the CSS plot (wide cumulative $\mathrm{AE}$ activity) at around 15:35 which corresponds to the attainment of the maximum load (Fig. 5). CSS plot, thus, appears as a reliable indicator of damage onset.

\subsection{Historic index}

Another aggregated measure of the acoustic energy released during the damage process is represented by the historic index. Historic index is the ratio between the average SS in the last events and the average SS during the all history. As described in Section 2.2, the variable $K$, which represents the event number from which computing the numerator of $H$, depends on three constants $C_{1}, C_{2}$ and $C_{3}$ varying with the number of hits and material type. Generally, it is assumed that $1-C_{3}=C_{I} / C_{2}$. In $[19,31]$ the values $C_{3}=0.8$ and $C_{I}=200$ are suggested (thus implying $C_{2}=1000$ ), with the rationale that the numerator should be the average signal strength of the last $20 \%$ or 200 hits, whichever is smaller. Given the low number of events recorded for the test setup described in this work, it was chosen to evaluate $K$ considering $C_{3}=0.8$ and $C_{I}=65$, hence $C_{2}=325$. 


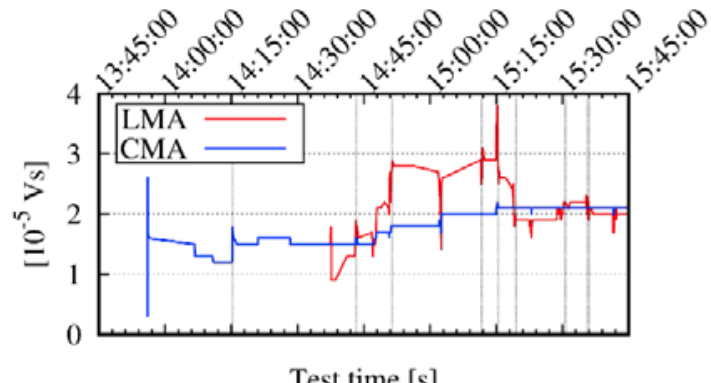

Fig. 8. Historic index: numerator and denominator evolution.

In Fig. 8, the evolution of numerator (last 65 events SS moving average, LMA) and denominator (cumulative SS moving average, CMA) is displayed. At the beginning $\left(N<C_{l}\right)$ the two terms are equal as per the definition of historic index. After that point, the two terms show differences, with CMA being clearly smoother than LMA. CMA becomes stable at 15:15, which corresponds to damage $D=87 \%$. On the other hand, any large scattering in LMA is a symptom of occurrence of events with SS markedly different from the previous history. Since average is sensitive to outliers, the occurrence of isolated energetic events leads to rapid increase and decrease of LMA, as for instance at 15:03 (compare this to Fig. 7, where the isolated energetic event is represented in the SS plot). Conversely, mainly monotonic LMA increase is detected between 14:39 and 14:50: comparing this trend to the history loading, it is possible to observe that in that phase AE activity is stably increasing due to the application of load not previously experienced by the specimen. After 15:15, a decrease and then stabilization of LMA is calculated. It is interesting to notice that the large step encountered in Fig. 7 at 15:35 for CSS does not have correspondence in the LMA plot of Fig. 8. This happens because LMA considers the last events regardless of their temporal distribution: the large increase of CSS plot is due to a large number of onaverage-energy events and not to low number of largeenergy events, as for instance at 15:03. The simultaneous use both indices may help identify these different cases. In the final stages of the test CMA and LMA reach similar values, meaning that the historic index is approaching unity. This trend was also observed in [19] in a corrosion test.

Finally, the historic index is reported in Fig. 9, in red line. The same historic index is then evaluated assuming as starting point 14:45, which represents approximately the middle of the test (with damage $D=67 \%$ ). This approach aims at investigating the feasibility of the historic index as parameter describing damage evolution even when the past history is not known, as for existing structures. In this case, the number of events considered is 325; all parameters $C_{1}, C_{2}, \mathrm{C} 3$ were left identical as in the previous analysis.

This preliminary analysis shows that at incipient collapse (from 15:18 onwards) the historic indices evaluated in both cases are similar and close to unity, even though the partial-history plot shows higher scattering due to reduced number of events.

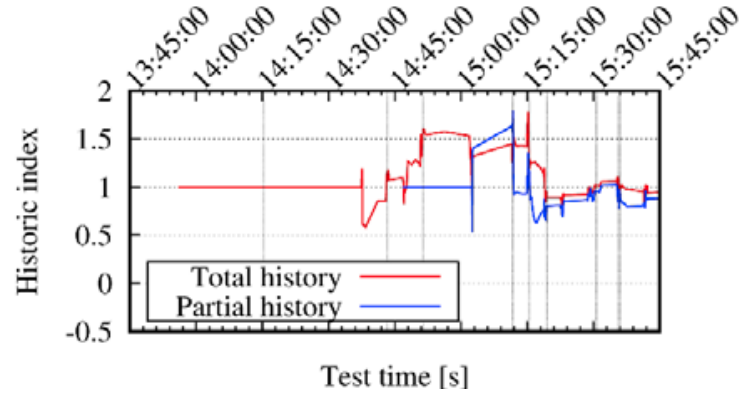

Fig. 9. Historic indices for total and partial history.

Even though definitive conclusions cannot be drawn at this stage, the use of partial-history historic index seems an interesting point which should be developed in future investigations.

\section{3 b-value}

As stated above, b-value represents the slope of the cumulative frequency plot of the magnitude distribution in logarithmic scale. Assuming the magnitude proportional to $A_{d B}$, the $b$-value may be obtained by multiplying the slope of amplitude cumulative frequency plot by 20 . If one wants to monitor the evolution of $b$ value with time, this evaluation must be carried out considering all events from the beginning to the instant of interest. The cumulative frequency plot of amplitude for five stages of the test are reported in Fig. 10.

It is possible to notice that at each stage the relationship between $\log N$ and $A_{d B}$ is almost linear only in the region comprised between 80 and $95 \mathrm{~dB}$. Furthermore, the slope of the quasi-linear region is not very much different in the different phases of the test, and this was also recognised in the tests reported in [24]. In the cited work, in which a shaking-table test was performed on a RC frame, large oscillation for the $b$ value was observed, with peaks and valleys that could be associated with instants of slow micro-cracking (low damage generation) and fast macro-cracking (high damage generation), respectively. For this reason, the authors suggested to revise the value 1.0 assumed as limit value for fully damaged specimen. In this work, given the behaviour reported in Fig. 10, no further analysis was then performed on b-value.

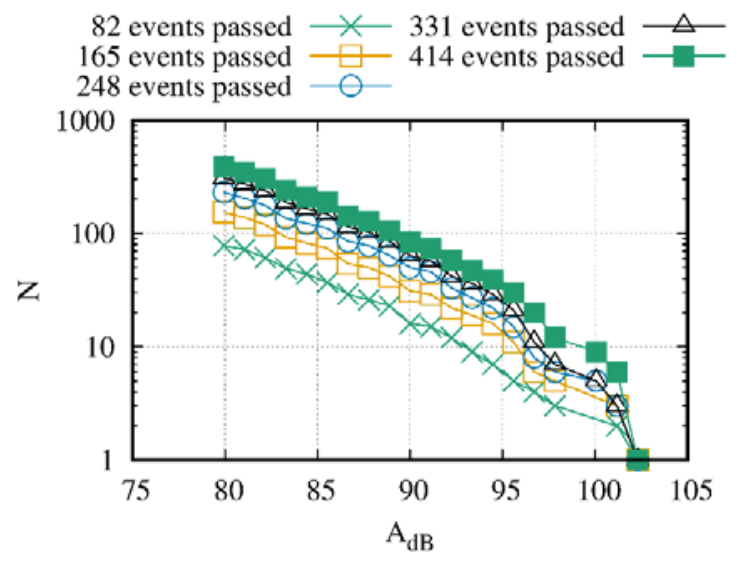

Fig. 10. Amplitude cumulative frequency at several stages. 


\section{Conclusions}

In this paper, damage assessment of Reinforced Concrete (RC) structures is studied by means of non-destructive techniques. In particular, Acoustic Emission (AE) analysis is adopted in a four-point bending test on a RC beam.

The main parameters reported in the specific literature have been resumed and evaluated on the data collected during the test. Interesting information were obtained, concerning the cracking status and the damage occurred.

The analysis of the Felicity ratio showed that this ratio drop down to $0.7-0.75$ when the damage surpasses a $60 \%$ threshold. The Signal Strength (SS) has also been analysed and compared with the Cumulative Signal Strength (CSS). It has been noticed that when the maximum load was attained, a large rise in the CSS occurred.

The historic index showed very interesting features, being a ratio of Last 65 events SS Moving Average (LMA) and Cumulative SS Moving Average (CMA). The variations of numerator and denominator have been analysed and plotted separately, in order to understand what happened approaching the crack of the specimen

Finally, the procedure to evaluate the $b$-value has been performed, but, since no evident variation were measured, the authors decided to postpone a detailed analysis on this parameter to further works.

In the end, it can be affirmed that the presented test and $\mathrm{AE}$ data analysis showed the possibility to help in assessing the damage of RC structures. Up to the authors opinion, the main challenge in this research domain will be the implementation and adjustment of wellestablished lab tests on specimen, to real and operating structures.

\section{References}

[1] M. Latour and G. Rizzano, Journal of Earthquake Engineering, 19, 1 (2015).

[2] M. Latour and G. Rizzano, Journal of Constructional Steel Research, 104 (2015).

[3] M. Latour, G. Rizzano, A. Santiago and D. Simoes, Journal of Constructional Steel Research, 101 (2014).

[4] P. Colajanni, L. La Mendola, M. Latour, A. Monaco and G. Rizzano, Journal of Constructional Steel Research, 111 (2015).

[5] Q. Dai, K. Ng, J. Zhou, E. L. Kreiger and T. M. Ahlborn, Construction and Building Materials, 31 (2012).

[6] A. Behnia, H. K. Chai and T. Shiotani, Construction and Building Materials, 65 (2014).

[7] M. Kaphle, Queensland University of Technology: PhD Thesis, 2012.

[8] S. Shahidan, R. Pulin, N. M. Bunnori and K. M. Holford, Construction and Building Materials, 45 (2013).
[9] M. Ohtsu, M. Uchida, T. Okamoto and S. Yuyama, ACI Structural Journal, 99, 4 (2002).

[10] NDIS-2421, Recommended Practice for In-Situ Monitoring of Concrete Structures by Acoustic Emission, Japanese Society for Non-Destructive Inspection, 2000.

[11] A. A. Pollock, International advances in nondestructive testing, 7 (1981).

[12] M. Ohtsu and H. Watanabe, Construction and Building Materials, 15, 5-6 (2001).

[13] S. Colombo, I. G. Main and M. C. Forde, J. Mater. Civ. Eng., 15, 3 (2003).

[14] J. H. Kurz, F. Finck, C. U. Grosse and H.-W. Reinhardt, Structural Health Monitoring, 5, 1 (2006).

[15] A. Carpinteri, G. Lacidogna and N. Pugno, Magazine of Concrete Research, 58, 9 (2006).

[16] A. Carpinteri, G. Lacidogna and S. Puzzi, Chaos, Solitons and Fractals, 41 (2009).

[17] I. Iturrioz, G. Lacidogna and A. Carpinteri, International Journal of Damage Mechanics, 23, 3 (2014).

[18] G. Birck, I. Iturrioz, G. Lacidogna and A. Carpinteri, Engineering Failure Analysis, 70 (2016).

[19] M. D. Benedetti, G. Loreto, F. Matta and A. Nanni, J. Mater. Civ. Eng., 26, 9 (2014).

[20] A. Abouhussien and A. Hassan, Structural Control and Health Monitoring, 24, 3, 2017.

[21] S. Colombo, M. Forde, I. Main and M. Shigeishi, Construction and Building Materials, 19, 10 (2005).

[22] A. R. Ridge and P. H. Ziehl, ACI Structural Journal, 103, 6 (2006).

[23] G. G. Clemeña, M. G. Lozev, J. C. J. Duke and M. F. J. Sison, Virginia Transportation Research Council, Charlottesville, Virginia (USA), 1995.

[24] F. Sagasta, A. Benavent-Climent, T. FernándezQuirante and A. Gallego, J Nondestruct Eval, 33 (2014).

[25] G. Ma and H. Li, Construction and Building Materials, 144 (2017).

[26] D. Li, F. Du and J. Ou, Smart Materials and Structures, 26 (2017).

[27] G. Ma, H. Li and Z. Duan, J. Compos. Constr., 16, 6 (2012).

[28] International Federation for Structural Concrete, Model Code 2010 - Final draft. fib 65, FIB, 2012.

[29] M. Ohtsu, M. Uchida, T. Okamoto and S. Yuyama, ACI Structural Journal, 99, 4 (2002).

[30] 212-ACD RILEM Technical Commitee, Materials and Structures, 43 (2010).

[31] S. C. Lovejoy, Final report SPR 633, Oregon Dept. of Transportation Bridge and Engineering Section,, Salem, OR (USA), 2008. 